\title{
Examination of Sexually Dimorphic Behavior on the Novel-Image Novel-Location Recognition Memory Test
}

\author{
Brian J. Piper ${ }^{1,2 * \#}$, Alia L. Yasen ${ }^{2 \#}$, Jeremy K. Miller ${ }^{2}$ \\ ${ }^{1}$ Department of Behavioral Neuroscience, Oregon Health \& Science University, Portland, USA \\ ${ }^{2}$ Department of Psychology, Willamette University, Salem, USA \\ E-mail: piperb@ohsu.edu,psy391@gmail.com \\ Received June 16, 2011; revised July 1, 2011; accepted July 9, 2011
}

\begin{abstract}
Objectives: Sex differences in object location memory favoring females appear to be a replicable phenomenon but may also depend on the task demands. This investigation evaluated if females outperformed males at both a short (immediate) and long (half-hour) interval between the learn and test condition using a recently developed version of the Novel-Image Novel-Location (NINL) test (Piper et al. 2011, Physiology \& Behavior, 103, 513 - 522). Methods: Young-adults $(\mathrm{N}=184)$ completed a standardized handedness inventory and the NINL. Results: Participants assigned to the Immediate and Delayed conditions did not differ in age, sex, or handedness. The NINL total score was higher among females at the Immediate, but not Delayed, interval. However, within the Delayed condition, females excelled at correctly identifying the unchanged items with a similar pattern for the Novel-Location (NL) scale. Conclusions: These findings are consistent with the view that sexually dimorphic performance favoring females in neurocognitive function can also extend to tasks that have a spatial component.
\end{abstract}

Keywords: Apolipoprotein E, Female, Learning, Memory

\section{Introduction}

Our understanding of the domains where there are sex differences in neurobehavioral function continues to be expanded and refined [1]. The standard view that women excel at verbal tasks and men at spatial tasks is likely an oversimplification as a female advantage has also been identified on selected measures that also have a spatial component [2]. One area that has repeatedly been shown as sensitive to sex differences is the Object Location Memory (OLM) in which participants are first instructed to learn a large set of common objects (e.g. an umbrella) and, on subsequent trials, identify which pairs of objects have switched positions $[3,4]$. The OLM has also been expanded to include a condition in which an object is moved to a formerly empty space. In contrast to the location-exchange, the location-shift condition did not show a female advantage [5], although see [6].

The Novel-Image Novel-Location (NINL) test contains some conceptual similarities to the OLM but is procedurally different as it is based on the rodent objectrecognition paradigm [7,8]. Participants view sets of

\#Both authors contributed equally. three unfamiliar pictures (learn images) and are subsequently asked to identify whether one of the three pictures has been replaced (the Novel-Image or NI condition) or relocated (the Novel-Location or NL condition [9]. In a broadly aged sample (6 to 86 ), we recently identified a significant female advantage on both NI and NL when the test was conducted immediately after learning the images [10]. Interestingly, the retention interval may be a key element in the detection of sex differences [11]. Therefore, the primary objective of the present report was to attempt to replicate and extend upon the sex difference observed in NINL using a more homogenous aged sample (young-adults) and at different intervals. As the NINL instrument [10] has undergone some modifications relative to an earlier version [9], a secondary goal is to complete a more refined analysis of each item and the correspondence with the total NINL scores.

\section{Methods}

\subsection{Procedures}

The Institutional Review Board at Willamette University 
approved all procedures. Subjects consisted of college students from a small private school receiving credit for experimental participation. Participants completed a questionnaire that queried about their age and sex and were asked which hand they used to complete activities. A Laterality Index was computed by adding up the number of activities (e.g. writing, drawing), completed with each hand and using the formula $[(\mathrm{R}-\mathrm{L}) /(\mathrm{R}+\mathrm{L})]^{*} 100$ [12]. A saliva sample was obtained for apolipoprotein E genotyping according to procedures outlined in [13].

The NINL, version 0.21 , was an extension of the previous NINL test [9]. The differences compared to version 0.1 were: 1) stimuli were imported into a slide show in Microsoft Power Point to precisely regulate the display time; 2 ) more detailed instructions and a practice trial so that the test could be more readily completed in a group setting; 3 ) the number of pictures was doubled to eighty with high resolution neutral images (e.g. a dustpan) from the International Affective Picture System [14]; and 4) the location of the changed item balanced across the four potential quadrants. Each slide was shown for $8.0 \mathrm{sec}$ during the learn phase. Either directly or approximately one-half hour after viewing these slides (henceforth referred to as Immediate or Delayed), testing commenced (eight NI, eight NL, and eight No-Change or NC). Participants in the Delayed condition completed other distracter tasks during the interval. The quadrant of the NI or NL was dispersed across the four quadrants with item type (NI, NL, or NC) staggered within the Learn and Test sets (see Table 1 for further details). Scoring consisted of $0-3$ points per NI/NL item with zero points awarded if the subject could not identify if a change had occurred, one point for correctly identifying that a change had happened, two points for also recognizing the change type (NI or NL), and three points for the previous plus accurately identifying the quadrant of the change. Correct identification of a $\mathrm{NC}$ item resulted in three points.

\subsection{Data-Analysis}

All statistics were performed using SPSS, version 16.0 (SPSS Inc., Chicago, IL) with an alpha level of 0.05. Pearson correlations were completed for each item score with the corresponding scale (NI, NL, or NC) and with the NINL total $(\mathrm{NI}+\mathrm{NL}+\mathrm{NC})$. Mean data is presented with the SEM. Cohen's $d$ was determined for group differences with 0.20 interpreted as small, 0.50 as medium, and 0.80 as a large effect size.

\section{Results}

The Immediate and Delayed groups did not differ based on age, sex, or handedness (Table 2). Table 3 shows the item to scale and item to total correlations for the 24item NINL instrument were all positive and significant. The item to scale associations were similar for the $\mathrm{NC}$ $(\mathrm{Min}=0.35$ to $\operatorname{Max}=0.63)$, NI $(0.32$ to 0.61$)$, and NL ( 0.30 to 0.59$)$ scales. The item to total NINL score correlations were generally homogenous for $\mathrm{NC}(0.21$ to $0.52)$ and NI (0.26 to 0.51$)$. Interestingly, the first NINL item also had the lowest correlation $(r=0.17)$ but values for the remaining seven NL items were higher $(r=0.29$ to 0.43 ). Further, the NC scale was moderately correlated with NI $(r(167)=0.39, p<0.0005)$ and NL $(r(169)=$ $0.39, p<0.0005)$ and NI and NL showed a similar association $(r(163)=0.37, p<0.0005)$.

Figure 1(a) shows that total NINL performance differed by both sex and retention interval. Females had higher scores than males in the Immediate $(d=0.59)$ but not Delayed condition. Females scored lower at the long interval relative to the Immediate $(d=0.47)$. Further analyses for each scale (Figure 1(b)) shows that sex, NINL difficulty, and task demands each determined recognition memory. Within the Immediate, males were

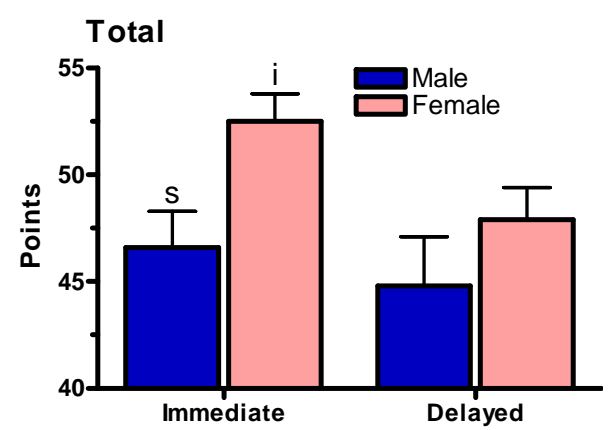

(a)



(b)

Figure 1. Novel-Image Novel-Location performance total (a) and for each scale (b) by sex and retention interval. NC: No-Change; NI: Novel-Image; NL: Novel-Location; ${ }^{\mathrm{s}} p<$ 0.05 sex difference within an interval; ${ }^{\mathrm{i}} p<0.05$ interval differences versus delayed; ${ }^{\mathrm{n}} p<0.05$ scale difference versus NC; ${ }^{1} p<0.05$ scale difference versus NL. 
significantly lower on NI $(d=0.59)$ but did not differ significantly on $\mathrm{NC}(t(104)=1.94, p=0.055, d=0.38)$ from females. In the Delayed, females outperformed males on $\mathrm{NC}(d=0.50)$ and showed a similar tendency for $\mathrm{NL}(t(72)=1.99, p=0.051, d=0.46)$. The NL was lower than both NC and NI independent of sex and interval.

\section{Discussion}

This report contributes to a very large and evolving literature on sex differences in neurocognitive function [1]. Clearly, the identification and direction of group differences based on sex depends on the sample size and nature of the task employed including how it is scored [2]. Males outperform females on the mental rotation test $[4,15]$ and exhibit almost equally large group differences on spatial navigation measures $[9,10,16]$. In contrast, less is definitely known about other domains. Although the NINL total was elevated among females only at the immediate interval, examination of each scale revealed a moderate effect size $(d \approx 0.50)$ for NI at the shorter period and on both NL and identifying the unchanged items at the longer period. This finding is broadly concordant with our previous study with a lifespan sample [10] and in line with a prior investigation with the OLM in which college aged females scored higher than males on identifying drawings of new objects $(d=0.46)$ and location exchanges $(d=0.44,[3]$. However, in the location shift of the OLM (the condition most similar to the NL), prior results have been contradictory with some [6], but not all [5], investigations identifying better performance among women.

In the OLM, participants view the original learn array and then, during repeated testing, may view elements of this same array again which makes these conditions difficult to compare directly. As Levy et al. [6] noted, this design could also introduce proactive interference. An advantage of the NINL is that this instrument has different learn sets that are subsequently tested in the NI, NL, or NC. Rodents find the location test much more challenging than the novel-object test [7]. Similarly, human participants did significantly less well on NL compared to NI at both intervals indicating that this is a robust phenomenon across species. The neural substrates that mediate these task specific processes have not yet been characterized on NINL but investigations with rats have shown that lesions of the perirhinal cortex impact object recognition memory [8]. It is tempting to speculate that location recognition would be more dependent on the dorsal stream (i.e. where) and image recognition on the dorsal ventral stream (i.e. what) structures although the integration of both occipto-parietal and occipito-temporal regions may also be essential [17].

The interval between the learn and test phases is a key element in task difficulty [18]. Adult female rats showed object-recognition at intervals ( 1 - 3 hours) that males could not. Conversely, males were capable of identifying when an object had been moved to a new position at several intervals that were too difficult for females [11]. A potential limitation is that only two intervals were examined in the present study. A slightly longer (1 hour) delay was evaluated during pilot testing and found to be unfeasible for this participant population. A comparison between an immediate and a 24-hour retention period produced the anticipated reduction at the longer-interval in a separate all-female sample [Thornburg \& Murphy, unpublished observations] but a multi-interval examination of sex differences should be a topic for further research.

A fundamental characteristic of any cognitive test is the type of the material to be learned. In addition to other caveats, this factor may also limit the generalizability between rodent and human recognition memory tests. Investigators utilizing laboratory animals can quite easily use objects that their subjects have never before experienced [18]. This frequently takes the form of children's toys [7] or commonplace household objects [19]. The NINL learn set (Table 1) consists of pictures from a database maintained for research purposes [14]. However, the images are of objects or scenes that can readily be labeled. Although the magnitude of sex differences in generalized verbal abilities is a matter of some contention [20], we suspect that females were more likely to employ verbal strategies to facilitate a deeper encoding of the image sets. Therefore, it is quite interesting that when abstract line drawings (which would limit the potential to label) are used as stimuli, there is conflicting data whether a female advantage is still detected [21-23]. Further, the Design Memory subtest of the Weschsler Memory ScaleIV includes a topographically more complicated NL type element with geometric shapes and abstract stimuli and sex differences are very minimal (personal communication from JM Laurer).

There are several future directions that may be worthy of some consideration for investigators interested in individual differences in NINL performance. A fascinating report determined that homosexual males substantially outperformed heterosexual males on Object-Location Memory [24]. Additional study with a more ethnically and socioeconomically diverse sample in which other information (e.g. sexual orientation, IQ, EEG) is obtained may prove fruitful as these factors could contribute to the present neurobehavioral profile.

Overall, there are clear sex differences on NINL which, when analyzed at the level of each scale, are independent 
Table 1. Images for the Novel-Image, Novel-Location test, Learn (L) and Test. Image numbers (in parentheses) are from the International Affective Picture System ([14]). Quadrants are north-west (NW), north-east (NE), south-east (SE) and south-west (SW) with empty quadrants depicted by a dash (-). Type is Novel Image (NI), Novel Location (NL) or No Change (NC).

\begin{tabular}{|c|c|c|c|c|c|c|c|c|}
\hline \multicolumn{5}{|c|}{ Learn } & \multicolumn{4}{|c|}{ Test } \\
\hline \multicolumn{2}{|l|}{ Slide } & \multicolumn{3}{|c|}{ Quadrant } & \multicolumn{4}{|c|}{ Slide } \\
\hline$\#$ & NW & $\mathrm{NE}$ & SW & SE & \# & Basis & Type & Description \\
\hline L01 & - & outlet (6150) & mushroom (5531) & train $(7039)$ & T01 & L07 & NL & $\operatorname{car}(8531)$ to NE \\
\hline L02 & dust pan (7040) & pocket watch (7190) & - & towel (7002) & T02 & L16 & $\mathrm{NC}$ & \\
\hline L03 & coffee cup (7057) & dark cloud (5594) & cube $(7185)$ & - & $\mathrm{T} 03$ & L04 & NI & clock (7211) at SE \\
\hline L04 & hammer (7034) & book (7090) & - & abstract painting (7830) & T04 & L11 & NI & crimps (7056) at NW \\
\hline L05 & yard (5130) & - & shoes $(7031)$ & woven basket (7010) & T05 & $\mathrm{L} 10$ & $\mathrm{NC}$ & \\
\hline L06 & set table (5849) & canyon (5661) & - & row boats $(5390)$ & T06 & L24 & NL & pins (7052) to NW \\
\hline L07 & hair dryer (7050) & - & convertible car (8531) & fan $(7020)$ & T07 & L05 & $\mathrm{NC}$ & \\
\hline L08 & - & leaves $(5750)$ & fork $(7080)$ & parking lot (7595) & T08 & L17 & NI & stool (7025) at SW \\
\hline L09 & fireworks (5480) & - & unlit light bulb (7055) & waste can (7060) & T09 & L18 & $\mathrm{NC}$ & \\
\hline L10 & - & satellites (5471) & blue cup (7009) & headlight (7095) & $\mathrm{T} 10$ & L02 & NL & pocket (7190) to SW \\
\hline L11 & rolling pin $(7000)$ & bus $(7140)$ & tiger lilly (5030) & - & $\mathrm{T} 11$ & L08 & NI & clothes (7242) at SW \\
\hline L12 & drill (7043) & latch (7059) & - & Native Amer pattern (7179) & $\mathrm{T} 12$ & L03 & NL & cube $(7185)$ to $\mathrm{SE}$ \\
\hline L13 & blue door (5731) & bridge (7547) & pink flower(1604) & - & $\mathrm{T} 13$ & L06 & $\mathrm{NC}$ & \\
\hline L14 & - & power lines (9080) & spoon (7004) & abstract painting (7161) & $\mathrm{T} 14$ & L15 & NL & bulb (7170) to SE \\
\hline L15 & lit bulb (7170) & ship (5395) & large baskets (7041) & - & $\mathrm{T} 15$ & L19 & $\mathrm{NC}$ & \\
\hline L16 & orchid (5010) & - & leaves $(5740)$ & empty pool (9360) & $\mathrm{T} 16$ & L09 & NI & rack (7217) at NW \\
\hline L17 & earth $(5890)$ & mountain top (5660) & airplane (7620) & - & $\mathrm{T} 17$ & L23 & NI & field (5250) at NW \\
\hline L18 & - & white bowl (7006) & tissue $(7950)$ & sports car $(8510)$ & $\mathrm{T} 18$ & $\mathrm{~L} 22$ & $\mathrm{NC}$ & \\
\hline L19 & clear glass (7035) & hydrant (7100) & gold bars $(8500)$ & - & $\mathrm{T} 19$ & L13 & $\mathrm{NC}$ & \\
\hline L20 & - & ferris wheel (7508) & orange flower (5020) & file cabinet (7705) & $\mathrm{T} 20$ & $\mathrm{~L} 20$ & NL & wheel (7508) to NW \\
\hline L21 & dumbells (7042) & - & lamp (7175) & yellow sail-boat $(8210)$ & $\mathrm{T} 21$ & L21 & NI & universe (5300) at SE \\
\hline L22 & shoes $(7038)$ & scarf (7205) & building (7491) & - & $\mathrm{T} 22$ & L12 & NL & Native (7179) to SW \\
\hline L23 & flowers $(5000)$ & semi-truck (7130) & - & freeway (7560) & $\mathrm{T} 23$ & L14 & NI & plate (7233) at NE \\
\hline L24 & - & clothes pins (7052) & umbrella (7150) & snow day (5635) & T24 & L01 & NL & train (7039) at NE \\
\hline
\end{tabular}

of the retention interval. The cognitive (e.g. attention or encoding) or biological (e.g. distinct neural substrates) mechanisms responsible for the female advantage on this and other similar tasks will be the subject of additional study. A strength of the NINL is that this procedure is based on the rodent object recognition test which may aid in translating the substantial knowledge base from rodents to humans.

\section{Acknowledgements}

Special thanks to Jacob Raber, PhD and Mark Stewart, $\mathrm{PhD}$ for their support and to Matthew B. Herson, Donna M. Nolan, Hannah M. Gandsey, Caitlin St. John, Casey 
Table 2. Participant characteristics by Novel-Image NovelLocation retention interval. apoE4: Apoliprotein E4 allele.

\begin{tabular}{ccc}
\hline & Immediate $(\mathrm{N}=106)$ & Delay $(\mathrm{N}=78)$ \\
\hline Age & $19.0(0.1)$ & $18.7(0.1)$ \\
Sex (\% Female) & $61.3 \%$ & $53.8 \%$ \\
apoE4+ & $21.7 \%$ & $17.9 \%$ \\
Laterality Index & $0.23(0.06)$ & $0.33(0.07)$ \\
\hline
\end{tabular}

Table 3. Pearson correlations for each item with the scale and total score (in parentheses) for the Novel-Image NovelLocation test. The $p$ value was $\leq \mathbf{0 . 0 0 1}$ for all correlations $\operatorname{except~}^{\mathrm{a} p}<0.05$.

\begin{tabular}{cccc}
\hline Item & No Change & Novel-Image & Novel-Location \\
\hline 1 & $0.51(0.41)$ & $0.61(0.46)$ & $0.30\left(0.17^{\mathrm{a}}\right)$ \\
2 & $0.50(0.40)$ & $0.32(0.33)$ & $0.46(0.43)$ \\
3 & $0.45(0.21)$ & $0.39(0.25)$ & $0.59(0.41)$ \\
4 & $0.48(0.36)$ & $0.50(0.31)$ & $0.53(0.35)$ \\
5 & $0.57(0.39)$ & $0.42(0.26)$ & $0.51(0.38)$ \\
6 & $0.35(0.28)$ & $0.58(0.51)$ & $0.51(0.37)$ \\
7 & $0.63(0.52)$ & $0.42(0.32)$ & $0.50(0.43)$ \\
8 & $0.41(0.35)$ & $0.60(0.47)$ & $0.47(0.29)$ \\
\hline
\end{tabular}

Conzatti, and Alan Curtis for assistance in data collection. Some of this data is also contained in the honor's thesis of ALY. This work was supported by the National Institute of Environmental Health Sciences (T32 ES00706031A1), and the National Institute of Drug Abuse (T32DA 07262 \& L30 DA027582-01).

\section{References}

[1] J. M. Andreano and L. Cahill, "Sex Influences on the Neurobiology of Learning and Memory," Learning and Memory, Vol. 16, 2009, pp. 248-66. doi:10.1101/lm.918309

[2] D. Voyer, A. Postma, B. Brake and J. ImperatoMcGinley, "Gender Differences in Object Location Memory: A Meta-Analysis," Psychonomic Bulletin and Review, Vol. 14, 2007, pp. 23-38. doi:10.3758/BF03194024

[3] I. Silverman, J. Choi and M. Peters, "The Hunter-Gatherer Theory of Sex Differences In Spatial Abilities: Data from 40 Countries," Archives of Sexual Behavior, Vol. 36, No. 2, 2007, pp. 261-268. doi:10.1007/s10508-006-9168-6

[4] J. Silverman and M. Eals, "Sex Differences in Spatial Abilities: Evolutionary Theory and Data" In: J. H. Barkow, L. Cosmides and J. Tooby, Eds., The Adapted Mind, Ox- ford, New York, 1992, pp. 534-549.

[5] T. W. James and D. Kimura, "Sex Differences in Remembering the Locations of Objects in an Array: Location-Shifts Versus Location Exchanges," Evolution and Human Behavior, Vol. 18, No. 3, 1997, pp. 155-163. doi:10.1016/S1090-5138(97)00004-4

[6] L. Levy, R. S. Astur and K. M. Frick, "Men and Women Differ in Object Memory but Not Performance of a Virtual Radial Maze," Behavioral Neuroscience, Vol. 119, No. 4, 2005, pp. 853-862. doi:10.1037/0735-7044.119.4.853

[7] S. L. Dix and J. P. Aggleton, "Extending the Spontaneous Preference Test of Recognition: Evidence of Object- Location and Object-Context Recognition," Behavioural Brain Research, Vol. 99, No. 2, 1999, pp. 191-200. doi:10.1016/S0166-4328(98)00079-5

[8] A. Ennaceur, "One-Trial Object Recognition in Rats and Mice: Methodological and Theoretical Issues," Behavioural Brain Research, Vol. 215, No. 2, 2010, pp. 244 255. doi:10.1016/j.bbr.2009.12.036

[9] A. M. Rizk-Jackson, S. F. Acevedo, D. Inman, D. Howieson, T. S. Benice and J. Raber, "Effects of Sex on Object Recognition and Spatial Navigation in Humans," Behavioural Brain Research, Vol. 173, No. 2, 2006, pp. 181190. doi:10.1016/j.bbr.2006.06.029

[10] B. J. Piper, S. F. Acevedo, K. R. Edwards, A. B. Curtiss, G. J. McGinnis and J. Raber, "Age, Sex, and Handedness Differentially Contribute to Neurospatial Function on the Memory Island and Novel-Image Novel-Location Tests," Physiology \& Behavior, Vol. 103, No. 5, 2011, pp. 513522. doi:10.1016/j.physbeh.2011.03.024

[11] J. S. Sutcliffe, K. M. Marshall and J. C. Neill, "Influence of Gender on Working and Spatial Memory in the Novel Object Recognition Task in the Rat," Behavioural Brain Research, Vol. 177, No. 1, 2007, pp. 117-125. doi:10.1016/0028-3932(71)90067-4

[12] R. C. Oldfield, "The Assessment and Analysis of Handedness: The Edinburgh Inventory," Neuropsychologia, Vol. 9, No. 1, 1971, pp. 97-113. doi:10.1016/0028-3932(71)90067-4

[13] F. Berteau-Pavy, B. Park and J. Raber, "Effects of Sex and APOE Epsilon 4 on Object Recognition and Spatial Navigation in the Elderly," Neuroscience, Vol. 147, No. 1, 2007, pp. 6-17. doi: 10.1016/j.neuroscience.2007.03.005

[14] P. J. Lang, M. M. Bradley and B. N. Cuthbert, "International Affective Picture System (IAPS): Affective Ratings of Pictures and Instruction Manual," Technical Report A-8 2008, University of Florida, Gainesville, FL.

[15] M. C. Linn, and A. C. Petersen, "Emergence and Characterization of Sex Differences in Spatial Ability: A Meta-Analysis," Child Development, Vol. 56, No. 6, 1985, pp. 1479-1498. doi:10.2307/1130467

[16] S. F. Acevedo, B. J. Piper, M. J. Craytor, T. S. Benice and J. Raber, "Apolipoprotein E4 and Sex Affect Neurobehavioral Performance in Primary School Children," Pediatric Research, Vol. 67, No. 3, 2010, pp. 293-299. doi:10.1203/PDR.0b013e3181cb8e68 
[17] R. Farivar, "Dorsal-Ventral Integration in Object Recognition," Brain Research Reviews, Vol. 61, No. 2, 2009, pp. 144-153. doi:10.1016/j.brainresrev.2009.05.006

[18] A. Ennaceur and J. Delacour, "A New One-Trial Test For Neurobiological Studies of Memory in Rats. 1: Behavioral Data," Behavioural Brain Research, Vol. 51, No. 1, 1988, pp. 47-59. doi:10.1016/0166-4328(88)90157-X

[19] B. J. Piper and J. S. Meyer, "Increased Responsiveness to MDMA in Adult Rats Treated Neonatally with MDMA," Neurotoxicology \& Teratology, Vol. 28, No. 1, 2006, pp. 95-102. doi:10.1016/j.ntt.2005.09.002

[20] J. S. Hyde and M. C. Linn, "Gender Differences in Verbal Ability: A Meta-Analysis," Psychological Bulletin, Vol. 104, No. 1, 1988, pp. 53-69. doi:10.1037/0033-2909.104.1.53

[21] J. Choi and N. L'Hirondelle, “Object Location Memory:
A Direct Test of the Verbal Memory Hypothesis," Learning \& Individual Differences, Vol. 15, 2005, pp. 237-245. doi:10.1016/j.lindif.2005.02.001

[22] L. Lejbak, M. Vrbanicic and M. Crossley, "The Female Advantage in Object Location Memory Is Robust to Verbalizability and Mode of Presentation of Test Stimuli," Brain \& Cognition, Vol. 69, No. 1, 2009, pp. 148-153. doi:10.1016/j.bandc.2008.06.006

[23] Q. Rahman, M. Bakare and C. Serinsu, "No Sex Differences in Spatial Location Memory for Abstract Designs," Brain \& Cognition, Vol. 76, No. 1, 2011, pp. 15-19. doi:10.1016/j.bandc.2011.03.012

[24] B. Hassan and Q. Rahman, "Selective Sexual OrientationRelated Differences in Object Location Memory, Behavioral Neuroscience, Vol. 121, 625-633. doi:10.1037/0735-7044.121.3.625 